\title{
EVOLUÇÃO DA GRAVIDEZ EM ADOLESCENTES MATRICULADAS NO SERVIÇO PRÉ-NATAL DO CENTRO DE SAÚDE GERALDO DE PAULA SOUZA, SÃO PAULO (BRASIL)
}

\author{
Arnaldo Augusto Franco de Siqueira* \\ Ana Cristina d'Andretta Tanaka* \\ Januário de Andrade ** \\ Pedro Augusto Marcondes de Almeida* \\ Solange Santiago*** \\ Clotilde Carolina Zanatelli **:* \\ Rosane Bier*** \\ Beatriz Machado de Souza Queirós*** \\ Maria Regina Saran***
}

\begin{abstract}
SIQUEIRA, A.A.F. de et al. Evolução da gravidez em adolescentes matriculadas no Strviço Pré-natal do Centro de Saúde Geraldo de Paula Souza. Sào Paulo (Brasil). Rev. Saúde públ., S. Paulo, 15:449-54, 1981.
\end{abstract}

RESUMO: Foram estudados 136 casos de gestações em mulheres abaixo de 20 anos de idade, matriculadas em um serviço de pré-natal. Foi constatada incidência significativamente mais elevada de prematuridade e de baixo peso ao nascer, quando os resultados foram comparados aos de um grupo de gestantes matriculadas no mesmo scrviço. Verificou-se ainda que o "status" sócio-econômico das gestantes adolescentes foi significativamente mais baixo. A incidência de cesáreas foi, também, significativamente menor, porém houve uma incidência maior de fórceps. O peso médio do recém-nascido foi significativamente menor na população estudada e, apesar de haver uma grande maioria de primigestas entre as adolescentes, a paridade não teve influência no peso do recém-nascido.

UNITERMOS: Gravidez na adolescência. Assistência pré-natal.

\section{INTRODUCYAO}

A adolescente é considerada por muitos autores como uma gestante de alto risco (Osofsky e Osofsky ${ }^{6}$, Duenhoelter e col. ${ }^{3}$ ). Esse maior risco a que a adolescente está exposta decorre, principalmente, de uma incidência maior de toxemia e desproporção céfalo-pélvica ${ }^{3}$.
Outros problemas têm sido relatados, especialmente nos EUA onde, anualmente, ocorrem mais de um milhão de gestações em adolescentes (Klein ${ }^{4}$, Rauh e col.i). Esses problemas dizem respeito a carências nutricionais, deficiência de cuidados pré-natais, ganho de peso excessivo, trabalho de parto

* Do Departamento de Saúde Materno-Infantil dr Faculdade de Saúde Pública da USP - Ar. Dr. Arnaldo, 715 - 01255 - São Paulo, SP - Brasil.

* Do Departamento de Prática de Saúde Pública da Faculdade de Saúde Pública da USP.

* Alunas de Nitrição da Facul lade de Ciências da Saúde São Camilo, estagiárias no Departamento de Saúde Materno-Infantil da. Faculdade de Saúde Pública da USP. 
SJQUEIRA, A.A.F. de et al. Evolução da gravidez em adolescentes matriculadas no Serviço Pré-natal do Centro de Saúde Geraldo de Paula Souza, São Paulo (Brasil). Rev. Saúde públ., S. Paulo, $15: 449-54,1981$.

prolongado, laceração de colo uterino, aumento de incidência de cesáreas e prematuridade, anemias e doenças venéreas (Ryan e Shneider ${ }^{8}$, Dott e Fort ${ }^{2}$ ).

Para o lado do concepto são várias as menções à incidência maior de recém-nascidos de baixo peso que, se sabe, está associada à maior mortalidade perinatal $e$ infantil.

Outros autores (Osofsky e Osofsky b) relatam aspectos associados à gravidez em adolescentes, dos quais avulta, mesmo em países desenvolvidos, a pobreza (problemas nutricionais, de higiene, de moradia e desajustes sociais).

Em nosso meio são escassas as referências ao assunto. Valente e col.10, analisando 610 mães adolescentes, em São Paulo (Brasil), encontraram $28 \%$ de mães com niveis pressóricos elevados, e $3,5 \%$ de mães com moléstias venéreas. Havia $10 \%$ de mães com 16 anos e menos; $75 \%$ eram primigestas e apenas $35 \%$ tinham recebido assistência pré-natal. Os autores referem ainda uma incidência de pramaturidade de $17,8 \%$, e uma incidência maior de lacerações de perineo e colo uterino.

Klein $*$ refere, nos EUA que o ambiente das adolescentes contribui para a atividade sexual precoce. Há um bombardeio constante de sexualidade com a televisão, o rádio, as canções, as publicações. $O$ sexo tem sido utilizado para vender toda classe de coisas, desde automóveis até ceras para assoalhos e sabōes em pó. Os programas de televisão mostram cenas sexuais e momentos de intimidade entre "pessoas muito belas". O fato e a fantasia, ainda segundo esse autor, são difíceis de separar na televisão e nos filmes.

Esse ambiente está presente em nosso meio o que, ao lado de um comportamento sexual menos inibido da sociedade em geral, poderá concorrer para que, no Brasil, a gravidez de adolescentes se torne uma preocupação cada vez maior.
Este trabalho pretende avaliar, retrospectivamente, a evolução da gravidez de adolescentes matriculadas no Serviço de Pré-natal do Centro de Saúde Geraldo de Paula Souza da Faculdade de Saúde Pública da Universidade de São Paulo (CS/FSP).

\section{MATERIAL E METODOS}

Foram selecionadas todas as gestantes inscritas entre $02 / 01 / 69$ e $31 / 12 / 78$ no Serviço Pré-natal do CS/FSP e que tivessem, na matricula, menos de 20 anos. Assim, a população de estudo foi constituída de 136 gestantes.

Foram estudadas as seguintes variáveis: idade, status sócio-econômico ${ }^{1}$, idade da menarca, número de gestações, incidência de óbitos neonatais, incidência de natimortos, tipo de parto, idade gestacional, peso do recém-nascido ao nascer, incidência de baixo peso ao nascer (peso igual ou inferior a $2.500 \mathrm{~g}$ ), dosagem de hemoglobina.

Para efeito de comparação foram analisados dados de uma população de gestantes sadias, matriculadas no mesmo serviço, e que foram objeto de estudo anterior ${ }^{\circ}$.

As gestantes adolescentes foram subdivididas em 2 subgrupos, de acordo com a literatura, ou seja, de 16 anos e menos e de 17 a 19 anos.

\section{RESULTADOS E DISCUSSAO}

Inicialmente foram confrontados os subgrupos: gestantes de 16 anos e menos e gestante de 17 a 19 anos. Nenhuma das diferenças encontradas foram estatísticamente significativas, pelo que se optou por estudar as adolescentes num grupo único.

Passou-se, a seguir, a comparar os resultados do grupo em estudo, com o das gestantes sadias matriculadas no mesmo serviço, que desempenhará doravante o papel de grupo controle. 
SIQUEIRA, A.A.F. de et al. Evolução da gravidez em adolescentes matriculadas no Serviço Pré-natal do Centro de Saúde Geraldo de Paula Souza, São Paulo (Brasil). Rev. Saúde públ., S. Paulo, $15: 449-54,1981$.

T A B E L A 1

Crianças recém-nascidas, segundo tipo de gestantes e peso ao nascer, de mães matriculadas no Centro de Saúde Geraldo de Paula Souza, no período de 02/01/69 a 31/12/78.

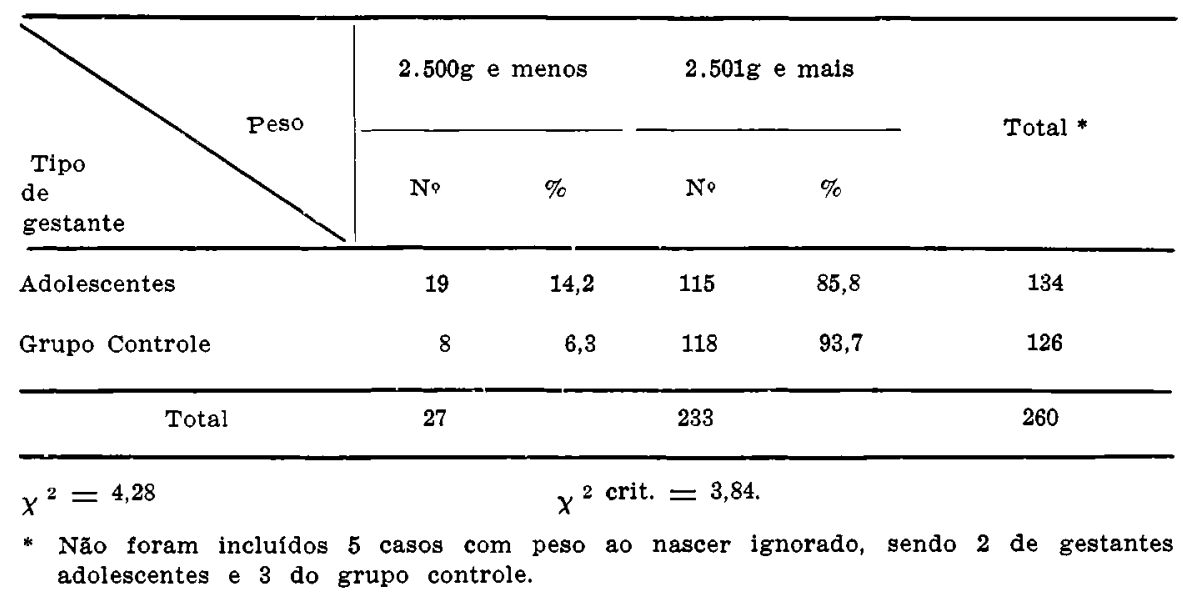

$\mathrm{Na}$ Tabela 1 verifica-se uma elevada incidência de recém-nascidos de baixo peso $(14,2 \%)$, comparada a $6,3 \%$ do grupo controle.

$\mathrm{Na}$ Tabela 2 verifica-se que a incidência de prematuridade é maior $(19,9 \%)$ entre as adolescentes que no grupo controle $(6,3 \%)$.
A Tabela 3 mostra que o peso médio do recém-nascido é significativamenté menor que o do grupo controle. Estes dados estão de acordo com o que referem a maioria dos autores, como Osofsky e Osofsky ${ }^{6}$, Klein ${ }^{4}$, Rauh e col. ${ }^{7}$, Ryan e Shneider ${ }^{8}$ e Dott e Fort ${ }^{2}$.

\section{T A B E L A 2}

Inci tência de prematuridade segundo tipo de gestantes matriculadas no Centro de Saúde Geraldo de Paula Souza e idade gestacional, no período de 02/01/69 a 31/12/78.

\begin{tabular}{l|lllll}
\hline & $\begin{array}{c}36 \text { semanas } \\
\text { e menos }\end{array}$ & & $\begin{array}{c}37 \text { semanas } \\
\text { e mais }\end{array}$ & Total \\
$\begin{array}{l}\text { Tipo } \\
\text { ge } \\
\text { gestante }\end{array}$ & No & $\%$ & No & $\%$ & \\
\hline $\begin{array}{l}\text { Adolescentes } \\
\text { Grupo Controle * }\end{array}$ & 27 & 19,9 & 109 & 80,1 & 136 \\
\hline \multicolumn{1}{c}{ Total } & 8 & 6,3 & 118 & 93,7 & $126 *$ \\
\hline
\end{tabular}

* Foram exci- ́dos 3 casos com idade gestacional ignorada.

$x^{2}$ obs $=10,30 \quad x^{2 \text { crítico }}=3,84$. 
SJQUEIRA, A.A.F. de et al. Evolução da gravidez em adolescentes matriculadas no Serviço Pré-natal do Centro de Saúde Geraldo de Paula Souza, São Paulo (Brasil). Rev. Saúde públ., S. Paulo, $15: 449-54,1981$.

\section{T A B E L A 3}

Crianças recém-nascidas, segundo tipo de gestantes e peso médio ao nascer, de mães matriculadas no Centro de Saúde Geraldo de Paula Sorza, no período de $02 / 01 / 69$ a $31 / 12 / 78$.

\begin{tabular}{|c|c|c|c|}
\hline $\begin{array}{l}\text { Tipo de } \\
\text { Gestante }\end{array}$ & Peso médio & Desvio padrāo & No de casos * \\
\hline Adolescentes & $\begin{array}{c}3.158 \mathrm{~g} \\
\mathrm{a}\end{array}$ & 627,67 & 134 \\
\hline Grupo controle & $3.291 \mathrm{~g}$ & 643,69 & 126 \\
\hline
\end{tabular}

$a=$ diferença encontrada significativa ao nível de $5 \%$.

* Foram excluidos 5 casos com peso ao nascer ignorado, sendo 2 de gestantes adolescentes e 3 do grupo controle.

$\mathrm{T}$ obs $=1.69 \quad \mathrm{t}$ crit. $=1,66$.

Pittaluga e col., citados por Valente e col. ${ }^{10}$ afirmam que a adolescente não é um problema obstétrico e, em se falando de idade mais favorável para a gestação, não se deve assinalar um limite inferior.

Talvez a incidência maior de baixo peso e prematuridade tenha relação com condições de vida mais do que com alguma deficiência para a maternidade, advinda da baixa idade. A maioria dos autores citados associam a gravidez em adolescentes à baixa condição social e econômica.

A Tabela 4 mostra que, mesmo para uma população relativamente homogênea como é a que freqüenta o CS/FSP, as adolescentes apresentaram níveis de status sócio-econômico significativamente mais baixos que as do grupo controle. Assim, quase $80 \%$ das adolescentes estão situadas nos niveis $1 \mathrm{e}$ 2 de status (baixo e médio-baixo), ao passo que apenas $21 \%$ situam-se nos niveis 3 e mais $(17 \%$ nível 3 e $3,5 \%$ nivel 4$) .0$ grupo controle apresentou $24 \%$ no nivel 3 , $8,5 \%$ no nivel 4 e $0,8 \%$ no nivel 5 , com um total de $33,3 \%$ para os niveis 3 e mais.

Este resultado favorece a hipótese de que as diferenças encontradas de peso de recém-nascido possam ser creditadas, pelo menos em parte, à condição sócio-econômica desfavorável como, aliás, a maioria dos autores também consideram.

\section{T A B E L A 4}

Gestantes matriculadas no Centro de Saúde Geraldo de Paula Souza, segundo o nível de status sócio-econômico, no período de 02/01/69 a 31/12/78.

Nínel de status
$\begin{gathered}\text { Sócio- } \\ \text { de }\end{gathered}$
gestante


SIQUEIRA, A.A.F. de et al. Evolução da gravidez em adolescentes matriculadas no Serviço Pré-natal do Centro de Saúde Geraldo de Paula Souza, São Paulo (Brasil). Rev. Saúde públ., S. Paulo, $15: 449-54,1981$.

Quanto ao tipo de parto, os resultados são diferentes dos da literatura, uma vez que a incidência de cesáreas em adolescentes foi significativamente menor que a do grupo controle (Tabela 5). Houve também uma incidência maior de fórceps, verificada também por Valente e col.10. Como a adoles- cente apresenta maior incidência de desproporção céfalo-pélvica, seria de esperar uma proporção maior de partos operatórios, o que efetivamente ocorreu, pois o $\chi^{2}$ observado para a soma de forceps e cesáreas, em relação a partos normais, foi igual a 5,95 para um $\chi^{2}$ crítico de 3,84 .

T A B E L A 5

Gestantes matriculadas no Centro de Saúde Geraldo de Paula Souza, segundo o o tipo de parto, no período de $02 / 01 / 69$ a $31 / 12 / 78$.

\begin{tabular}{|c|c|c|c|c|c|c|}
\hline \multirow{2}{*}{$\begin{array}{l}\text { Tipo } \\
\text { de } \\
\text { gestante }\end{array}$} & \multicolumn{2}{|c|}{ Normal (1) } & \multicolumn{2}{|c|}{ Forceps (2) } & \multicolumn{2}{|c|}{ Cesárea (3) } \\
\hline & No & $\%$ & No & $\%$ & No & $\%$ \\
\hline Adolescentes * & 92 & 68,1 & 24 & 17,8 & 19 & 14,1 \\
\hline Grupo Controle & 68 & 52,7 & 11 & 8,5 & 50 & 38,8 \\
\hline
\end{tabular}

$\chi^{2}$ para tipo de parto 1 e $2=1,04$.

$\chi^{2}$ para tipo de parto 2 e $3=14,47$.

$\chi^{2}$ para tipo de parto 1 e $3=16,15 \chi^{2}=$ crítico $=3,84$.

* Foi excluído 1 caso pois não havia informação sobre o tipo de parto.

Como a maioria das gestantes adolescentes são primigestas (neste trabalho $67,9 \%$ das adolescentes eram primigestas), e como o primeiro filho é menor que os demais, procurou-se verificar se no presente caso a paridade pudesse estar influindo nos resultados. A Tabela 6 mostra que não houve diferença significativa no peso do recém-nascido, apesar de que os filhos das primigestas tiveram um peso médio menor que os demais.

T A B E L A 6

Peso ao nascer de filhos de adolescentes, conforme fossem ou não primigestas.

\begin{tabular}{lccc}
\multicolumn{1}{c}{ Paridade * } & Média & Desvio padrão & No de casos \\
\hline $\begin{array}{l}\text { Primigesta } \\
\begin{array}{l}\text { Secundigesta } \\
\text { e mais }\end{array}\end{array}$ & $3.136,26$ & 563,71 & 91 \\
\hline t obs $=0,47$ & $3.197,67$ & 758,18 & 43 \\
em 2 casos a paridade era ignorada. & t crit. $=0,66$.
\end{tabular}

A idade média na menarca das adolescentes foi de 12,5 anos, de acordo com os dados da literatura. Assim, Valente e col.10 citam 13 anos, e Nelson ${ }^{5}$ refere, nos EUA, 12,7 anos.

O estudo desse grupo de adolescentes permitiu verificar que as principais dife- renças da gestação em adolescentes são a incidência maior de prematuridade e o menor peso do recém-nascido. Este dado é da maior importância quando se leva em conta a maior morbidade e mortalidade a que estão sujeitas essas crianças.

O fato de só terem sido levantados, no 
SIQUEIRA, A.A.F. de et al. Evolução da gravidez em: adolescentes matriculadas no Serviço Pré-natal do Centro de Saúde Geraido de Paula Souza, São Paulo (Brasil). Rev. Saúde públ., S. Paulo, $15: 449-54$, 1981 .

presente trabalho, 136 casos em 10 anos, não serve como base da magnitude do problema, pois sabe-se que grande parte das gestantes adolescentes não procura qualquer serviço de pré-natal. Freqüentemente o parto é o primeiro contato da paciente com alguma agência de saúde. É o que citam Valente e col.10 e Ryan e Shneider:.

\section{CONCLUSOES}

As gestantes adolescentes matriculadas no Serviço Pré-natal do Centro de Saúde
Geraldo de Paula Souza apresentaram, quando comparadas com um grupo controle, incidência maior de prematuridade, maior proporção de recém-nascidos de baixo peso $e$ indice de status sócio-econômico mais baixo, bem como uma incidencia menor de cesáreas e maior de fórceps.

Ao serem mantidas as atuais tendências de "liberação" do comportamento reprodutivo, a gestação em adolescentes tem tudo para se transformar em pouco tempo, em nosso meio, em um problema de Saúde Pública.

SIQUEIRA, A.A.F. de et al. [Evolution of pregnancy in adolescent mothers at the pre-natal clinic of the Health Centre Geraldo de Paula Souza, S. Paulo (Brazil)]. Rev. Saúde públ., S. Paulo, 15:449-54, 1981.

ABSTRACT: A hundred and thirty six cases of pregnancy in women below the age of twenty, patients of a pre-natal clinic, were studied. There was found to be a significantly higher rate of premature births and of low-birth-weight, in comparison with a group of normal pregnant women, treated at the same clinic. It was also found that the socio-economic status of the pregnant teen-agers was considerably lower. The rate of cesarian sections was also considerably lower, but the rate of forceps was higher. The average weight of the newborn children was considerably lower in the population sample studied and despite there being a great majority of primipara among the adolescents, the factor parity had no influence on the weight of the newborn.

UNITERMS: Pregnancy in adolescence. Pre-natal care.

\section{REFERENCIAS BIBLIOGRÁFICAS}

1. ALVARENGA, A.T. de et al. Indice de status sócio-econômico da família da mulher grávida que frequienta o Centro de Saúde Geraldo de Paula Souza da Faculdade de Saúde Pública da Universidade de São Paulo. Rev. Saúde públ., S. Paulo, 7:351-61, 1973.

2. DOTT, A.B. \& FORT, A.T. Medical and social factors affecting early teenage pregnancy. Amer. J. Obstet. Gynec, 125 :532-4, 1976.

3. DUENHOELTER, J.H. et al. Pregnancy performance of patients under fifteen years of age. Obstet. and Gynec., 46:49-52, 1975.

4. KLEIN, L. Antecedentes del embarazo en adolescentes. Clin. Obstet. Gynec., 21:1198-208, 1978.

5 NELSON, R.M. Correlaciones fisiológicas de la puberdad. Clin. Obstet. Gynec. 21 : 1185-97,1978.
6. OSOFSKY, J.D. \& OSOFSKY, H.J. Embarazo en la seginda década de la vida. consideraciones psicosociales. Clin. Obstet. Gynec., 21:1209-23, 1978.

7. RAUH, J.L. et al. The reproductive adolescent. Pediat. Clin. N. Amer, 20:1005$-20,1973$

8. RYAN, G.M. \& SHNEIDER, J.M. Complicaciones obstétricas en adolescentes. Clin. Obstet. Gynec., 21:1243-9, 1978.

9. SIQUEIRA, A.A.F. de Estudo de um conjunto de curvas antropométricas no diagnóstico de estado nutricional de gestantes e sua relacão com o tamanho do recém-nascido, São Paulo, 1979. [Tese de Doutoramento - Faculdade de Saúde Pública da USP].

10. VALENTE, C.A. et al. Assistência pré e perinatal à mãe adolescente. J. bras. Ginec. $83: 228-35,1977$.

Recebido para publicaçāo em 17/09/1980 Aprovado para publicaşão em 21/07/1981 